\title{
Effects of hypercapnia and arterial hypotension and hypertension on cerebrospinal fluid pulse pressure and intracranial volume-pressure relationships
}

\author{
C J J AVEZAAT, J H M VAN EIJNDHOVEN, AND D J WYPER \\ From the Departments of Neurosurgery and Electroneurology, Academic Hospital \\ Rotterdam-Dijkzigt, Erasmus University, Rotterdam and the Department of Clinical Physics, \\ Institute of Neurological Sciences, Glasgow
}

SUMMARY In twelve anaesthetised, ventilated dogs the effects of hypercapnia and pharmacologically induced arterial hypotension and hypertension on the interrelation between volumepressure response (VPR) and cerebro-spinal fluid (CSF) pulse pressure were studied during continuous inflation of a supratentorial extradural balloon. Hypercapnia did not significantly affect the intracranial volume-pressure relationships, but did cause a significant increase in gradient of the relationship between CSF pulse pressure and intracranial pressure (ICP). Alteration of the arterial blood pressure showed opposite effects on VPR and CSF pulse pressure. A decrease in VPR and an increase in pulse pressure were observed during arterial hypotension; the reverse was found during arterial hypertension. The discrepancy between the effects on VPR and CSF pulse pressure of the variables under study was explained by changes in the transient increase in cerebral blood volume per cardiac cycle. On the basis of the results of this study it will be possible, during clinical ICP monitoring, to interpret changes in the CSF pulse pressure to ICP ratio in terms of changes in intracranial volume-pressure relationships.

$\begin{array}{ll}\text { Definitions of abbreviations and symbols } \\ \text { ICP } & \text { Intracranial pressure } \\ \text { VFP } & \text { Ventricular fluid pressure } \\ \text { SAP } & \text { Systemic arterial pressure } \\ \Delta \text { SAP } & \text { Systemic arterial pulse pressure } \\ P_{\text {eq }} & \text { Equilibrium pressure, physiological steady } \\ & \text { state ICP } \\ \Delta P & \text { CSF pulse pressure or VPR } \\ \text { V } & \text { Change in total volume of the craniospinal } \\ & \text { compartment } \\ \Delta V & \text { Injected volume during a volume pressure } \\ & \text { test or the transient increase in CBV per } \\ \text { CBV } & \text { cardiac cycle } \\ \text { VPT } & \text { Verebral blood volume } \\ \text { VPR } & \text { Volume-pressure test } \\ \mathrm{E} & \text { Elastance of the craniospinal compartment } \\ \mathrm{E}_{1} & \text { Elastance coefficient } \\ \mathrm{CBF} & \text { Cerebral blood flow } \\ \mathrm{HR} & \text { Heart rate } \\ \mathrm{PaCO} & \text { Arterial carbon dioxide tension }\end{array}$

Address for reprint requests: Dr CJJ Avezaat, Department of Neurosurgery, Academic Hospital Rotterdam, DIJK2IGT; Dr Molewaterplein 40, 3015 GD Rotterdam, The Netherlands.

Accepted 15 August 1979
In previous clinical and experimental studies $^{12}$ the interrelation between the cerebrospinal fluid (CSF) pulse pressure and the volume-pressure response (VPR) was established. These studies had been designed to investigate whether the CSF pulse pressure could be used as a continuous parameter of the intracranial volume-pressure relationships during clinical intracranial pressure (ICP) monitoring. The height of the CSF pulse was shown to be determined by two factors: (a) the slope of the intracranial volume-pressure curve and $(b)$ the magnitude of the transient increase in cerebral blood volume (CBV) per cardiac cycle $(\Delta V)$. During experimental brain compression in dogs this volume change was shown to be constant up to a mean ICP of $60 \mathrm{~mm} \mathrm{Hg}$ and we consequently concluded that changes in the CSF pulse pressure to ICP ratio, occurring below this pressure, truly reflect changes in the slope of the volume-pressure curve. In the pressure range above $60 \mathrm{~mm} \mathrm{Hg}$ $\Delta \mathrm{V}$ was found to increase progressively. The origin of the transient increase in CBV was attributed to the interaction between the cerebral 

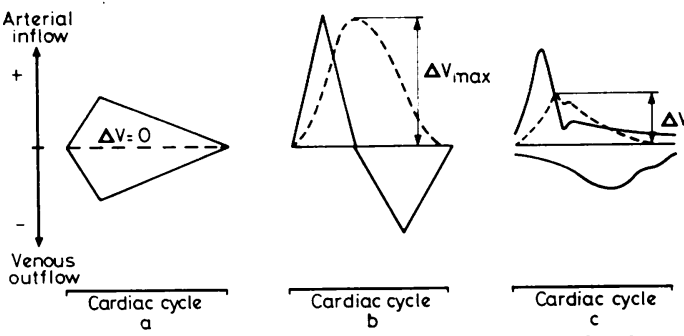

Fig 1 Concept of transient increase in cerebral blood volume per cardiac cycle $(\Delta V)$. The cerebral arterial inflow and venous out flow curves are schematically drawn; the change in cerebral blood volume is indicated by the dashed curve. The magnitude of $\Delta V$ is determined by the interaction between inflow and outflow. If both flow profiles were identical, the resulting volume change would be nil (a). If inflow and outflow were completely separated, $\Delta V$ would be maximal (b). Inflow and outflow, however, have different pulsatile flow patterns, thus producing an intermediate volume change $(c$.

arterial inflow and venous outflow of blood during a cardiac cycle (fig 1). One of the main determinants of these flow profiles is the cerebrovascular resistance, and the increase in $\Delta \mathrm{V}$ above the ICP of $60 \mathrm{~mm} \mathrm{Hg}$ was therefore explained by failure of cerebral blood flow (CBF) autoregulation and progressive vasomotor paralysis.

Factors influencing cerebral hemodynamics, such as the arterial carbon dioxide tension $\left(\mathrm{PaCO}_{2}\right)$ and the systemic arterial pressure (SAP), may be expected to affect $\Delta \mathrm{V}$ and consequently the height of the CSF pulse. Hypercapnia is known to increase the pulse pressure. ${ }^{345}$ This eff ect, however, is rather complex, as hypercapnia also produces a rise in ICP and SAP. Conflicting results have been reported with regard to the effects of changes in SAP on the CSF pulse pressure. Hamer et $a^{5}$ found an increase of the pulse height during arterial hypertension. An increased transmission of pulse from cerebral artery to vein, implying a consequent increase of the CSF pulse pressure, was observed during hypotension by Symon. ${ }^{4}$ Moreover, with regard to the effect on the CSF pulse pressure, clear distinction is often not made between the level of SAP and the height of the arterial pulse. The blood pressure has also been claimed to influence the intracranial elastance, ${ }^{67}$ which constitutes another mechanism through which the CSF pulse pressure may be affected.

Critically ill patients are exposed to changes in both $\mathrm{PaCO}_{2}$ and SAP. Because of the complex effects of these variables on the CSF pulse pressure and the intracranial elastance, the role of the pulse pressure in the assessment of the in- tracranial volume-pressure relationships during clinical ICP monitoring tends to be obscured. For this reason an experimental study was designed in dogs to establish the effects of hypercapnia as well as anterial hypotension and hypertension on the interrelation between the CSF pulse pressure and the VPR as a measure of intracranial elastance during rising ICP produced by an expanding extradural balloon.

\section{Methods}

Twelve anaesthetised, ventilated adult mongrel dogs of both sexes (body weights 14 to $28 \mathrm{~kg}$ ) were studied. The anaesthesia, surgical preparation, and methods of continuously recording ventricular fluid pressure (VFP), cisterna magna pressure, SAP, and central venous pressure have been described previously. ${ }^{2}$ After all pressures had stabilised an extradural balloon in the right frontal region was gradually distended by infusion of normal saline at a rate of $1 \mathrm{ml} / 40$ minutes. As a measure of the elastance the $\mathrm{VPR}^{8}$ was used. At regular intervals throughout the experiment clusters of four volume-pressure tests (VPT) each were carried out by rapid injections of $0.05 \mathrm{ml}$ of normal saline into the lateral ventricle. The VPR was calculated from the immediate change in mean VFP. To reduce variability the mean value of the four VPTs of each cluster was taken as the VPR for that interval and related to the mean preinjection VFP. Eight CSF pulse pressures were calculated by taking the average value of the pulse pressures over the last respiratory cycle before and the first cycle after each VPT. The means of the four pulse pressures before and after each test were related to the mean preinjection and postinjection VFP respectively. As a result of this procedure each time interval yielded one VPR and two pulse pressures. Arterial blood gases were measured throughout the study using a direct reading electrode system (Radiometer BMS 3) and the readings were corrected for temperature differences between the animal and the recording system. ${ }^{\circ}$

After conclusion of the experiments the animals were killed and the brains removed. After weighing (weights between 71 and $102 \mathrm{~g}$ ) the brains were fixed in formalin and thereafter sectioned coronally at $5-\mathrm{mm}$ intervals. The correct position of the VFP recording needles was verified and major brain lesions other than those produced by the expanding balloon were excluded.

The twelve animals were divided into two 
groups of six each. In the first group the effects of hypercapnia and in the second those of arterial hypotension and hypertension on the CSF pulse pressure and the VPR were studied. The experimental design was such that each animal served as its own control.

Protocol hypercapnia Brain compression was started at normocapnia. Hypercapnia was produced by administering $5 \%$ carbon dioxide to the inspired gases for five minutes, after which period a steady state was obtained as indicated by an infrared analyser monitoring the end-tidal $\mathrm{CO}_{2}$. During the following 15 minutes the animal was allowed to stabilise at a normal normocapnic level. At time 0 and 5 minutes volume-pressure tests were done and blood gases taken. The same manoeuvre was repeated at intervals of 20 minutes until the VFP approached the level of the blood pressure, at which time the experiment was terminated. In this way VPRs and CSF pulse pressures were obtained at normocapnic $\left(\mathrm{PaCO}_{2}=\right.$ $\mathrm{M} \pm \mathrm{SD}=40.9 \pm 2.7 \mathrm{~mm} \mathrm{Hg}$ ) and hypercapnic $\left(\mathrm{PaCO}_{2}=57 \cdot 8 \pm 3 \cdot 3 \mathrm{~mm} \mathrm{Hg}\right)$ levels throughout the period of brain compression.

Protocol arterial hypotension and hypertension Brain compression was started at arterial normotension. SAP was reduced over 5 minutes by intravenous titration of a $0.1 \%$ solution of trimethaphan (Arfonad). For the next 5 minutes SAP was increased by continuous intravenous infusion of angiotensin (Hypertensin-CIBA, $3 \mu \mathrm{g} /$ $\mathrm{ml}$ ). Thereafter the animal was allowed to stabilise at a normotensive level for 10 minutes. VPTs were performed at 0,5 , and 10 minutes. The same procedure was repeated every 20 minutes. In this way VPRs and CSF pulse pressures were obtained throughout the period of brain compression at arterial normotension $(\mathrm{M} \pm \mathrm{SD}=135.5 \pm 5.9 \mathrm{~mm}$ $\mathrm{Hg}$ ), hypotension $(89.0 \pm 5 \cdot 2 \mathrm{~mm} \mathrm{Hg})$, and hypertension $(175 \cdot 3 \pm 9 \cdot 2 \mathrm{~mm} \mathrm{Hg}) . \mathrm{PaCO}_{2}$ was maintained within normal limits $(37-43 \mathrm{~mm} \mathrm{Hg})$ during this experiment.

Data analysis The mathematical model used to analyse the data of this study has been extensively described and experimentally verified in a previous study. ${ }^{2}$ The intracranial volume-pressure relationship was defined by a monoexponential function:

$$
\mathbf{P}=\mathbf{P}_{\mathrm{rq}} \mathbf{e}^{\mathrm{E}_{1} \mathrm{~V}}
$$

Where $P=I C P, P_{c q}=a$ constant equal to the ICP at the equilibrium point-that is, the normal, physiological steady state ICP $-E_{1}=$ the elastance coefficient, a constant defining the slope of the volume-pressure curve, and $V=$ the change in total volume of the craniospinal compartment. From this equation the relationships of both VPR and CSF pulse pressure to the ICP were derived:

$$
\Delta \mathbf{P}=\mathbf{P}\left[\mathrm{e}^{\mathrm{E}_{1} \Delta \mathrm{V}}-1\right]
$$

where $\Delta P=V P R$ or $C S F$ pulse pressure and $\Delta \mathrm{V}=$ the injected volume during a VPT $(0.05 \mathrm{ml})$ as well as the transient increase in CBV per cardiac cycle. For both VPR and pulse pressure this was demonstrated to be a linear function up to a break point occurring at a mean ICP of $60 \mathrm{~mm} \mathrm{Hg}$. $E_{1}$ and $\Delta V$ were therefore concluded to be constant below the break point. These parameters can be calculated from the slopes of the VPR and CSF pulse pressure versus ICP regression lines using equation 2, which can then be rewritten as:

$$
E_{1}=\frac{1}{\Delta V} \ln \left[\frac{\Delta P}{P}+1\right]
$$

where $\frac{\Delta \mathbf{P}}{\mathbf{P}}=$ the slope of the VPR versus ICP regression line and $\Delta \mathrm{V}=$ the injected volume during a volume-pressure test, and :

$$
\Delta \mathrm{V}=\frac{1}{\mathrm{E}_{1}} \ln \left[\frac{\Delta \mathrm{P}}{\mathrm{P}}+1\right]
$$

where $\Delta V=$ the transient increase in $C B V$ per cardiac cycle, $\Delta \mathrm{P} / \mathrm{P}=$ the slope of the CSF pulse pressure versus ICP regression line and $\mathrm{E}_{1}=$ the elastance coefficient calculated from equation 3 .

Above the break point the VPR remained constant, implying a constant elastance (E) and thus a linear volume-pressure relationship :

$$
\mathrm{E}=\frac{\Delta \mathbf{P}}{\Delta \mathrm{V}}=\text { constant and } \mathbf{P}=\mathrm{EV} \text {. }
$$

Since the pulse pressure continued to increase linearly with the ICP, we concluded that this was caused by a progressive increase in the volume change per cardiac cycle.

\section{Results}

EFFECTS OF HYPERCAPNIA ON THE VOLUMEPRESSURE RESPONSE AND THE CSF PULSE PRESSURE Figure 2 illustrates, in a single animal, the 


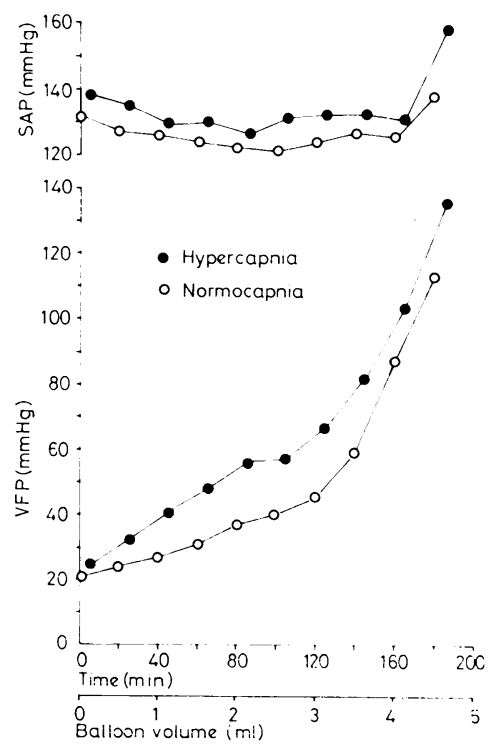

Fig 2 Ventricular fluid pressure (VFP) and systemic arterial pressure $(\mathrm{SAP})$ at normocapnia (Mean $\mathrm{PaCO}_{2}$ $( \pm S \mathrm{D}) 36 \cdot 3 \pm 2 \cdot 7 \mathrm{~mm} \mathrm{Hg})$ and at hypercapnia $\left(\mathrm{PaCO}_{2}=\right.$ $53.8 \pm 5.4 \mathrm{~mm} \mathrm{Hg}$ ) during steady rate inflation ( $1 \mathrm{ml} / 40 \mathrm{~min}$ ) of an extradural balloon in a single animal. Hypercapnia produced progressively larger rises in VFP. Above VFP of $45 \mathrm{~mm} \mathrm{Hg}$, however, $\mathrm{CO}_{2}$ response decreased.

effect of hypercapnia on the ventricular fluid pressure during gradual inflation of the extradural balloon. Hypercapnia produced a progressive increase in VFP, but at a particular level of ICP the $\mathrm{CO}_{2}$ response started to

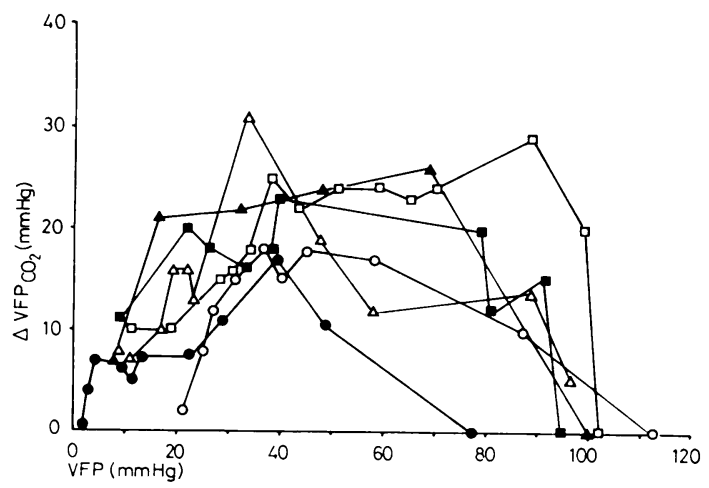

Fig 3 Increase in ventricular fluid pressure in each time interval produced by hypercapnia $\left(\triangle V F P_{\mathrm{Co} 2}\right.$; see also figl2) against VFP during cerebral compression in si $x$ dogs. Average increase in VFP reached its maximum around $V F P$ of $50 \mathrm{~mm} \mathrm{Hg}$ corresponding with the break point pressure in the relationship beteen volume-pressure resf n'se and VFP $(53.5 \mathrm{~mm} \mathrm{Hg}$; see text $)$.

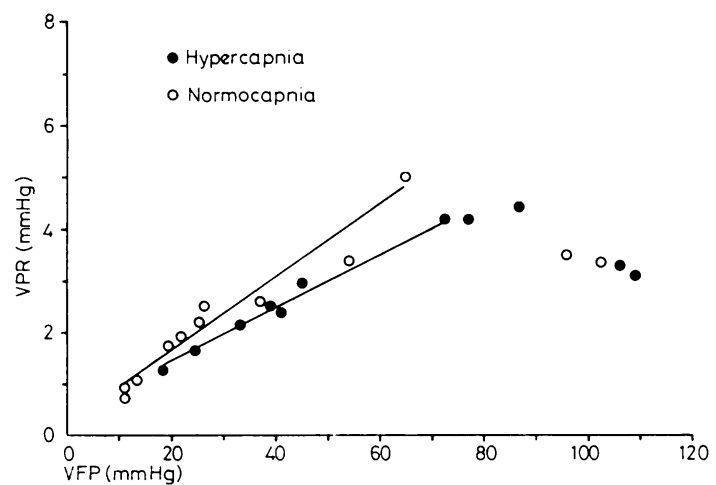

Fig 4 Plot of the volume-pressure response (VPR) against the ventricular fluid pressure (VFP) during hypercapnia (mean $\mathrm{PaCO}_{2}( \pm S \mathrm{SD}) 60 \cdot 7 \pm 4 \cdot 3 \mathrm{~mm} \mathrm{Hg}$ ) compared with normocapnia $\left(\mathrm{PaCO}_{2}=43.6 \pm 2.9 \mathrm{~mm} \mathrm{Hg}\right)$ in single animal. A linear relationship is shown up to a breakpoint above which the VPR slightly decreases. Regression lines are shown: $y=0.07 x+0.3(r=0.97, p<0.001)$ for normocapnia and $y=0.05 x+0.5(r=0.99, p<0.001)$ for hypercapnia. Gradients of the regression lines are not significantly different (F test).

decrease and finally $\mathrm{CO}_{2}$ could no longer increase the VFP. This is clearly demonstrated when the increase in VFP, produced by hypercapnia in each time interval, is plotted against the VFP at normocapnia (fig 3).

During both normocapnia and hypercapnia the VPR increased linearly with rising VFP until a breakpoint was reached above which the VPR levelled off and sometimes slightly decreased (fig 4). Close to the level of the blood pressure the VPR started to increase again, but this pressure range was not further studied. The "normocapnic" breakpoint occurred at a mean VFP of $53.4 \mathrm{~mm} \mathrm{Hg}$ (range $42.8-64.6 \mathrm{~mm} \mathrm{Hg}$ ) and the "hypercapnic" break point at a VFP of $60.8 \mathrm{~mm} \mathrm{Hg}$ (range $47.8-72 \mathrm{~mm} \mathrm{Hg}$ ). The difference in break point VFP was statistically significant (Wilcoxon matched-pairs test : $\mathrm{p}<0.05)$. Below the breakpoint significant linear relationships between VPR and VFP were always obtained $(p<0 \cdot 05)$. Consequently the monoexponential volume-pressure model could be applied. The elastance coefficients $\left(E_{1}\right)$ were calculated from the slopes of the regression lines using equation 3 and the results are given in table 1. Hypercapnia produced a decrease of $E_{1}$, indicating a flattening of the volume-pressure curve, in five animals and an increase of $E_{1}$ in one, but none of these differences attained statistical significance ( $F$ test applied to the slopes of the regression lines). 
Table 1 Elastance coefficient $\left(E_{1}\right)$ and transient increase in cerebral blood volume per cardiac cycle $(\Delta V)$ below and elastance and increase in $\Delta V$ above the break point, arterial carbon dioxide tension $\left(\mathrm{PaCO}_{z}\right)$, systemic arterial pressure (SAP), systemic arterial pulse pressure $(\triangle S A P)$, and heart rate $(H R)$ during normocapnia and hypercapnia. Values are means $\pm S D$

\begin{tabular}{|c|c|c|c|c|c|c|c|c|}
\hline $\begin{array}{l}\text { Animal } \\
\text { no }\end{array}$ & $\begin{array}{l}E_{1} \\
(1 / m l)\end{array}$ & $\begin{array}{l}\Delta V \\
(m l)\end{array}$ & $\begin{array}{l}\mathrm{PaCO}_{2} \\
(\mathrm{~mm} \mathrm{Hg})\end{array}$ & $\begin{array}{l}S A P \\
(m m H g)\end{array}$ & $\begin{array}{l}\Delta S A P \\
(m m H g)\end{array}$ & $\begin{array}{l}H R \\
\text { beats/min }\end{array}$ & $\begin{array}{l}\text { Elastance } \\
(\mathrm{mm} \mathrm{Hg} / \mathrm{ml})\end{array}$ & $\begin{array}{l}\text { Increase in } \\
\Delta V(m l / m m ~ H g)\end{array}$ \\
\hline \multicolumn{9}{|c|}{ Normocapn:a } \\
\hline 1 & 1.5 & 0.113 & $36.3 \pm 2.7$ & $126.5 \pm 5.0$ & $58.1 \pm 4.5$ & $136.7 \pm 23.0$ & 98 & 0.0023 \\
\hline 2 & 1.9 & 0.059 & $43.0 \pm 2.8$ & $135.1 \pm 7.3$ & $54.3 \pm 6.3$ & $70.7 \pm 8.2$ & 110 & 0.0015 \\
\hline$\overline{3}$ & 1.4 & 0.036 & $43.6 \pm 2.9$ & $145.8 \pm 7.4$ & $52.2 \pm 5.6$ & $105.8 \pm 14.2$ & 68 & 0.0021 \\
\hline 4 & 1.4 & 0.077 & $39.7 \pm 0.8$ & $132.4 \pm 4.0$ & $44.6 \pm 5.2$ & $83.3 \pm 7.5$ & 88 & 0.0017 \\
\hline 5 & 1.2 & 0.074 & $40.4 \pm 1.9$ & $166.3 \pm 4.7$ & $71.0 \pm 3.3$ & $91.0 \pm 9.9$ & 82 & 0.0028 \\
\hline 6 & 1.2 & 0.142 & $42.4 \pm 2.4$ & $147.7 \pm 2.7$ & $62.1 \pm 4.1$ & $87.0 \pm 4.2$ & 76 & 0.0047 \\
\hline Mean & 1.4 & 0.084 & $40.9 \pm 2.7$ & $142.3 \pm 14.3$ & $57.1 \pm 9.0$ & $95.8 \pm 23.1$ & 87 & 0.0025 \\
\hline \multicolumn{9}{|c|}{ Hypercapnia } \\
\hline 1 & 1.4 & 0.200 & $53.8 \pm 5.4$ & $131.2 \pm 3.3$ & $65.9 \pm 2.8$ & $133.8 \pm 19.5$ & 104 & 0.0027 \\
\hline 2 & 2.1 & 0.071 & $61.2 \pm 4.0$ & $161.1 \pm 10.6$ & $65.8 \pm 6.2$ & $72.4 \pm 5.4$ & 114 & 0.0018 \\
\hline 3 & 1.0 & 0.098 & $60.7 \pm 4.3$ & $155.4 \pm 8.1$ & $57.4 \pm 4.7$ & $108.4 \pm 7.1$ & 84 & 0.0014 \\
\hline 4 & 1.0 & 0.107 & $60.4 \pm 3.1$ & $143.8 \pm 3.5$ & $47.1 \pm 3.9$ & $91.0 \pm 6.2$ & 80 & 0.0020 \\
\hline 5 & 0.8 & 0.200 & $55.6 \pm 2.5$ & $182.5 \pm 5.6$ & $77.2 \pm 4.1$ & $93.0 \pm 8.5$ & 94 & 0.0024 \\
\hline 6 & 1.0 & 0.220 & $55.3 \pm 3.3$ & $152.1 \pm 6.1$ & $63.6 \pm 2.9$ & $81.0 \pm 3.0$ & 80 & 0.0043 \\
\hline Mean & 1.2 & 0.149 & $57.8 \pm 3.3$ & $154.4 \pm 17.3$ & $62.8 \pm 10.0$ & $96.6 \pm 21.9$ & 93 & 0.0024 \\
\hline
\end{tabular}

The ICP range above the break point was in view of the levelling-off phenomenon of the VPR (and in agreement with the results of a previous study ${ }^{2}$ ) conceived as a pressure range

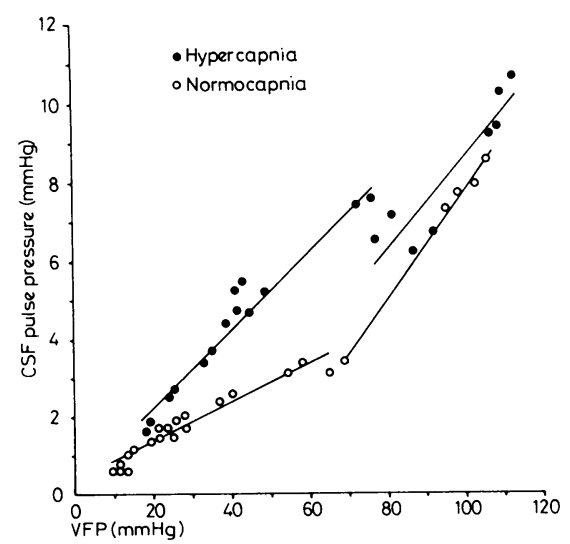

Fig 5 Effect of hypercapnia on relationship between CSF pulse pressure and ventricular fluid pressure (VFP) in single animal (same animal as in fig 4.) Regression lines are shown for pressure ranges on both sides of break points in VPR-VFP relationships. Below break point CSF pulse pressure increased significantly faster $(F$ test, $p<0.001)$ during hypercapnia $(y=0 \cdot 10 x+0 \cdot 2, r=0 \cdot 97, p<0 \cdot 001)$ than during normocapnia $(y=0.05 x+0.4, r=0.97$, $p<0.001)$. Above break points gradients of relationships at hypercapnia $(y=0 \cdot 12 x-3 \cdot 3, r=0 \cdot 92, p<0 \cdot 001)$ and at normocapnia $(y=0.14 x-6 \cdot 3, r=1 \cdot 00, p<0.001)$ were not significantly different. When slopes above break points were compared with those of below break points, significant change was observed during normocapnia only (Ftest, $p<0.001$ ). with a constant elastance to which therefore a linear volume-pressure model should be applied. The elastance (E) was calculated from the mean VPR using equation 5 (table 1). In none of the animals did hypercapnia produce a significant change in the high pressure elastance.

A typical example of the effect of hypercapnia on the relationship between CSF pulse pressure and VFP is shown in fig 5. All the animals behaved in this way. In the analysis of the pulse pressure data the same break points were taken into account as were found in the VPR-VFP relationships. On either side of the break point there was a significant positive linear correlation ( $\mathrm{p}$ always $<0.001$ ) between CSF pulse pressure and VFP, but the effect of hypercapnia was different. Below the break point the gradient of the hypercapnic regression line was always steeper compared to normocapnia. In five animals the difference attained statistical significance ( $\mathrm{F}$ test : $\mathrm{p}<0.05$ in one and $\mathrm{p}<0 . \mathrm{CO1}$ in four). Above the break point, however, the effect of $\mathrm{CO}_{2}$ on the pulse pressure was less pronounced. In four animals the slope of the hypercapnic relationship was only slightly steeper, in one it was weaker, whereas in the remaining animal there was no difference in slope between hypercapnia and normocapnia. None of these differences were statistically significant. The break point was operative in still another way. During normocapnia the pulse pressure always increased more rapidly above than below the break point, the difference in gradient of the regression lines attaining 


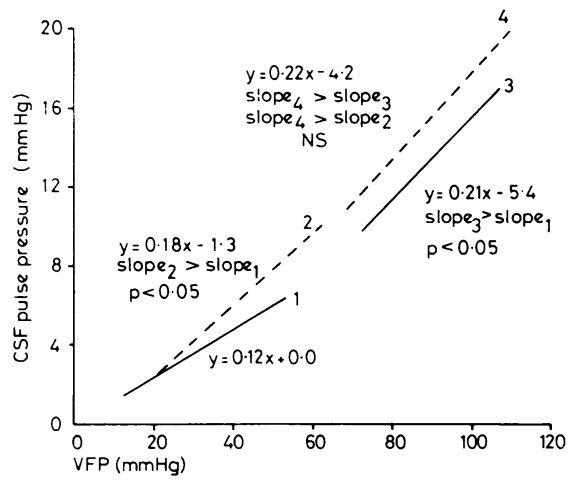

Fig 6 Composite graph of effect of hypercapnia on relationship between CSF pulse pressure and ventricular fluid pressure (VFP) during cerebral compression in six dogs. Mean regression lines and corresponding regression equations are shown for hypercapnia (dashed line) and normocapnia (uninterrupted line) on both sides of break points. Difference in gradient between various relationships was statistically evaluated by applying Wilcoxon's matched-pairs test to the regression lines of individual animals.

statistical significance in five animals ( $F$ test: $\mathrm{p}<0.05$ in two and $\mathrm{p}<0.001$ in three). During hypercapnia the pulse pressure showed a significantly steeper rise above the break point in two animals only ( $F$ test : $p<0.05$ and $\mathrm{p}<0.001)$. These results are summarised in fig 6 , where the mean regression lines of the whole group of animals are given. According to the analytical model the linear correlations of both VPR and CSF pulse pressure with the VFP below the break point imply a constant change in $\mathrm{CBV}$ per cardiac cycle $(\Delta \mathrm{V})$. The magnitude of $\Delta \mathrm{V}$ was calculated from the slopes of the regression lines using equation 4 . In each animal hypercapnia produced a significant increase in the intracranial volume change per cardiac cycle (table 1). The rapid increase of the pulse pressure above the break point can, in view of the constant elastance in this pressure range, be caused only by a progressive increase in $\Delta \mathrm{V}$. The increase in $\Delta \mathrm{V}$ per $\mathrm{mm} \mathrm{Hg}$ was calculated using equation 5 and the results are given in table 1 , showing no significant differences between normocapnia and hypercapnia.

Hypercapnia also produced a slight rise in blood pressure as well as in arterial pulse pressure (table 1). This should be taken into account in discussing the effects of hypercapnia on both VPR and pulse pressure. The heart rate remained unchanged.
EFFECTS OF ARTERIAL HYPOTENSION AND HYPERTENSION ON THE VOLUME-PRESSURE RESPONSE AND THE CSF PULSE PRESSURE The hypotensive, normotensive, and hypertensive levels of the SAP in the various animals together with the corresponding arterial pulse pressures ( $\triangle$ SAP) and heart rates (HR) are given in table 2. The typical reactions of the ventricular fluid pressure to the administration of trimethaphan and angiotensin are shown in fig $7 a$ and $b$. The VFP first changed concomitantly with the SAP, but generally after 10-30 seconds the VFP began to move into the opposite direction. This was considered to be the effect of a vasomotor response to the change in cerebral perfusion pressure consisting of vasoconstriction and vasodilatation respectively. So long as this response was effective the net effect on the ICP was small. At a certain level of ICP, however, the response started to diminish and finally disappeared, and thereafter the VFP closely followed the SAP (fig 8).

When the VPR was plotted against the VFP for all three levels of SAP the same type of relationship was found as in the previous series: a linear ( $p$ always $<0.01$ ) increase of the

Table 2 Elastance coefficient $\left(E_{l}\right)$, transient increase in cerebral blood volume per cardiac cycle $(\Delta V)$, systemic arterial pressure (SAP), systemic arterial pulse pressure $(\triangle S A P)$, heart rate $(H R)$ during arterial normotension, hypotension, and hypertension. Values are means $\pm S D$

\begin{tabular}{|c|c|c|c|c|c|}
\hline$\underset{\text { no }}{\text { Animal }}$ & $\begin{array}{l}E_{1} \\
(1 / m l)\end{array}$ & $\begin{array}{l}\Delta V \\
(m l)\end{array}$ & $\begin{array}{l}S A P \\
(m m H g)\end{array}$ & $\begin{array}{l}\Delta S A P \\
(m m H g)\end{array}$ & $\begin{array}{l}H R \\
\text { beats/min }\end{array}$ \\
\hline $\begin{array}{l}1 \\
2 \\
3 \\
4 \\
5 \\
6\end{array}$ & $\begin{array}{l}1.4 \\
0.8 \\
1.0 \\
1.9 \\
1.9 \\
1.7\end{array}$ & $\begin{array}{l}0.090 \\
0.062 \\
0.050 \\
0.055 \\
0.016 \\
0.017\end{array}$ & $\begin{array}{c}\text { Normotension } \\
129.1 \pm 7.8 \\
132.6 \pm 7.4 \\
140.2 \pm 5.6 \\
145.1 \pm 7.0 \\
133.6 \pm 6.0 \\
132.6 \pm 3.6\end{array}$ & $\begin{array}{l}46.6 \pm 5.9 \\
32.8 \pm 4.9 \\
32.2 \pm 4.8 \\
54.5 \pm 3.0 \\
28.3 \pm 2.6 \\
26.5 \pm 8.0\end{array}$ & $\begin{array}{l}120.5 \pm 15.2 \\
157.3 \pm 7.7 \\
138.4 \pm 9.0 \\
143.3 \pm 9.0 \\
143.1 \pm 25.4 \\
135.5 \pm 21.6\end{array}$ \\
\hline Mean & 1.5 & 0.048 & $135.5 \pm 5.9$ & $36.8 \pm 11.2$ & $139.7 \pm 12.0$ \\
\hline $\begin{array}{l}1 \\
2 \\
3 \\
4 \\
5 \\
6\end{array}$ & $\begin{array}{l}1.4 \\
0.6 \\
1.0 \\
1.4 \\
1.4 \\
0.8\end{array}$ & $\begin{array}{l}0.084 \\
0.099 \\
0.134 \\
0.097 \\
0.036 \\
0.062\end{array}$ & $\begin{array}{c}\text { Hypotension } \\
87.9 \pm 8.7 \\
86.9 \pm 6.0 \\
90.0 \pm 5.4 \\
96.0 \pm 4.5 \\
92.5 \pm 6.2 \\
80.9 \pm 8.4\end{array}$ & $\begin{array}{l}39.3 \pm 6.4 \\
26.3 \pm 1.4 \\
28.4 \pm 1.8 \\
39.5 \pm 2.5 \\
29.1 \pm 2.9 \\
24.5 \pm 2.9\end{array}$ & $\begin{array}{l}123.3 \pm 12.5 \\
143.6 \pm 6.6 \\
114.5 \pm 4.0 \\
152.0 \pm 24.2 \\
124.8 \pm 17.0 \\
127.0 \pm 10.3\end{array}$ \\
\hline Mean & 1.1 & 0.085 & $89.0 \pm 5.2$ & $31.2 \pm 6.6$ & $130.9 \pm 14.0$ \\
\hline $\begin{array}{l}1 \\
2 \\
3 \\
4 \\
5 \\
6\end{array}$ & $\begin{array}{l}2.3 \\
1.2 \\
1.5 \\
1.7 \\
2.4 \\
1.5\end{array}$ & $\begin{array}{l}0.038 \\
0.025 \\
0.019 \\
0.066 \\
0.008 \\
0.006\end{array}$ & $\begin{array}{l}\text { Hypertension } \\
175.9 \pm 15.1 \\
164.7 \pm 6.1 \\
174.9 \pm 10.1 \\
190.5 \pm 3.6 \\
178.5 \pm 9.6 \\
167.3 \pm 8.0\end{array}$ & $\begin{array}{l}48.7 \pm 5.6 \\
27.8 \pm 6.3 \\
23.5 \pm 4.0 \\
49.9 \pm 5.8 \\
29.5 \pm 6.0 \\
22.7 \pm 3.1\end{array}$ & $\begin{array}{r}98.0 \pm 14.7 \\
156.8 \pm 13.1 \\
121.8 \pm 11.7 \\
129.9 \pm 7.7 \\
125.1 \pm 14.1 \\
132.0 \pm 5.6\end{array}$ \\
\hline Mean & 1.8 & 0.027 & $175.3 \pm 9.2$ & $33.7 \pm 12.4$ & $127.3 \pm 18.9$ \\
\hline
\end{tabular}



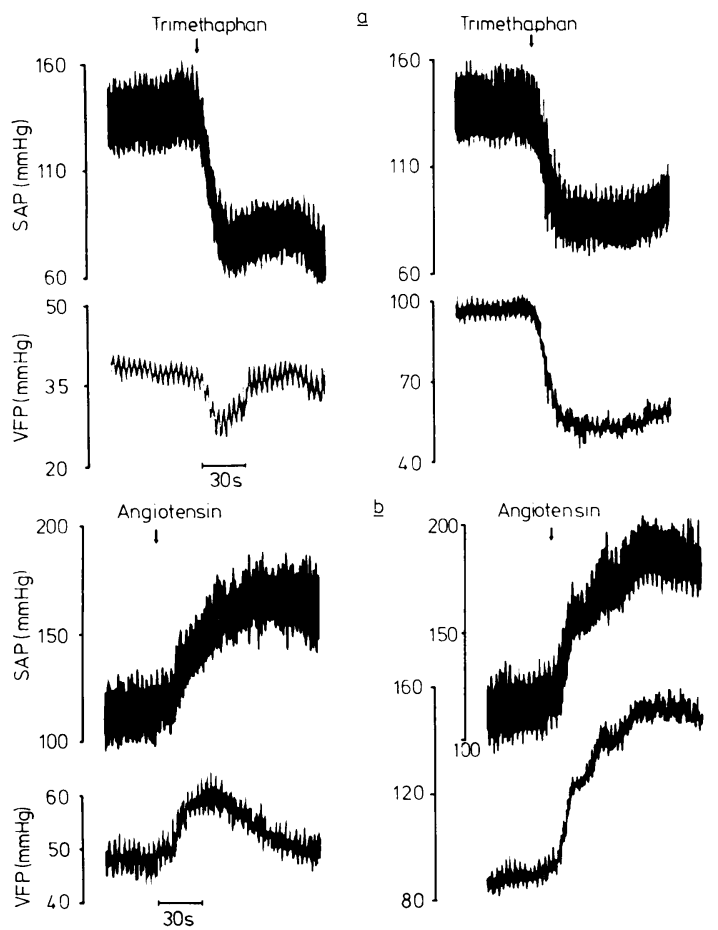

Fig 7 Tracings of systemic arterial pressure $(S A P)$ and ventricular fluid pressure (VFP) during intravenous administration of trimethaphan ( $a$ ) and angiotensin $(b)$ before (left) and after (right) the break point. Typical response of VFP to change in perfusion pressure before break point is believed to represent intact cerebrovascular reactivity. After break point VFP closely followed $S A P$, indicating impaired autoregulation.

VPR below and a constant VPR above a break point (fig 9). In all the animals an increase in VPR with rising SAP was observed. When the slopes of the regression lines at the three blood pressure levels were compared, a significant difference was found between normotension and hypotension in three animals $(\mathrm{p}<0 \cdot 01)$ and between normotension and hypertension in the other three animals $(p<0.05)$. Only when hypotension was compared with hypertension was a significant change in slope found in all the animals $(p<0.1$ in one, $p<0.05$ in two, and $\mathrm{p}<0.01$ in the remaining animals). The overall results, however, indicate a rise in VPR with increasing blood pressure, as shown in table 3 , where the mean regression lines are given. The elastance co-efficients were calculated from the slopes of the individual regression lines using equation 3 (table 2). Hypertension generally produced an increase in $E_{1}$ and hypotension a decrease, implying a steeper and a flatter intracranial volume-pressure curve respectively.

The break points occurred at a mean VFP of $45.7 \mathrm{~mm} \mathrm{Hg}$ (range $41.4-48.8 \mathrm{~mm} \mathrm{Hg}$ ) for hypotension, $55.1 \mathrm{~mm} \mathrm{Hg}$ (range $38.3-69.6 \mathrm{~mm}$ $\mathrm{Hg}$ ) for normotension, and $58.3 \mathrm{~mm} \mathrm{Hg}$ (range $45 \cdot 6-73.8 \mathrm{~mm} \mathrm{Hg}$ ) for hypertension. The break point pressures at hypotension and at hypertension were significantly different (Wilcoxon matched-pairs test, $\mathrm{p}<0.05)$. The corresponding cerebral perfusion pressures were $42.7 \mathrm{~mm} \mathrm{Hg}$, $79.6 \mathrm{~mm} \mathrm{Hg}$, and $119.8 \mathrm{~mm} \mathrm{Hg}$. The break point pressure at normotension corresponded with the VFP at which the vasomotor response disappeared (fig 8) and this was confirmed in each animal. In this series the ICP range above the break point was not further studied; too few data points were available as the SAP changes caused large fluctuations in ICP.

The plots of the CSF pulse pressure against VFP showed linear correlations for each blood pressure level ( $p$ always $<0.01$; fig 10 ). Comparison of the slopes of the regression lines yielded the reverse result, as was found in the

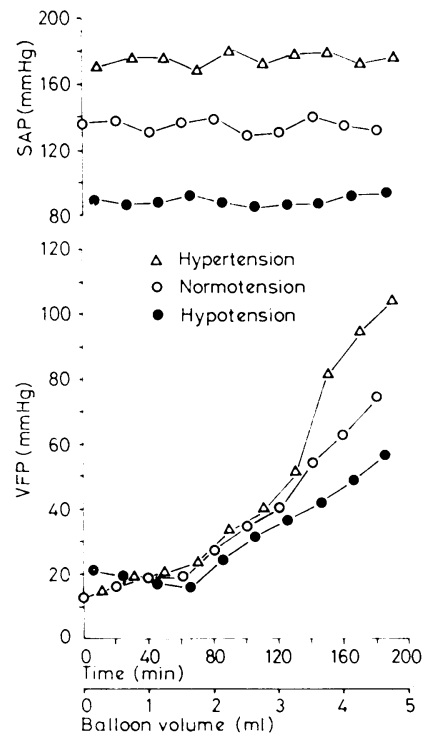

Fig 8 Composite plot of ventricular fluid pressure (VFP) and systemic arterial pressure (SAP) at arterial normotension and drug induced hypotension and hypertension during steady rate inflation $(1 \mathrm{ml} / 40 \mathrm{~min})$ of extradural balloon in six dogs. From mean VFP of $55 \mathrm{~mm} \mathrm{Hg}$ onwards changes in SAP produced significant changes in $V F P$. In every animal this event coincided with disappearance of vasomotor response ( fig $7 a, b)$ and with break point in VPR-VFP relationship. 


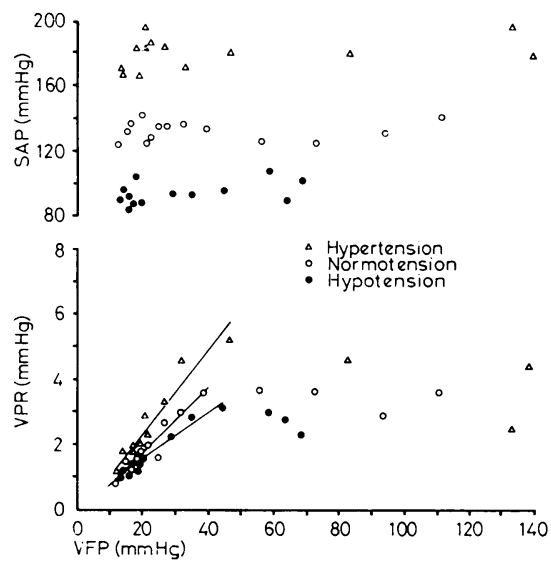

Fig 9 Effects of arterial hypotension and hypertension on relationship between volume-pressure response $(V P R)$ and ventricular fluid pressure (VFP) in single animal. Various levels of systemic arterial pressure $(S A P)$ are shown. At all three levels of $S A P$ a break point in relationship was observed. Below break point linear correlations were obtained with $y=0.10 x-0.2(r=0.96, p<0.001)$ for normotension, $y=0.07 x+0.3(r=0.98, p<0.001)$ for hypotension, and $y=0.13 x-0.2(r=0.95, p<0.001)$ for hypertension. The slopes were significantly different between hypotension and normotension and hypotension and hypertension (Ftest), $p<0.01$ ). Above break points $V P R$ remained constant or slightly decreased.

VPR against VFP plots: a decrease in slope with increasing SAP level (table 3). A significantly weaker slope at hypertension compared to normotension was found in five animals ( $F$ test, $\mathrm{p}<0.05$ ). In the remaining animal the slope was steeper at hypertension, but the difference was not significant. Hypotension produced a steeper slope than normotension in all but one animal, but in only three animals did the difference attain statistical significance ( $F$ test, $\mathrm{p}<0.05$ ). Only when hypertension was compared with hypotension was a uniform change in slope found in all the animals (F test, $\mathrm{p}<0.05$ in five animals). The transient change in CBV per cardiac cycle was calculated for each blood pressure level in each animal using equation 4 (table 2 ). The difference in $\Delta \mathrm{V}$ between the various blood pressure levels is larger than the difference in slope of the regression lines. The reason is that $\Delta \mathrm{V}$ is also proportional to the inverse of $E_{1}$, and $E_{1}$ is, in contrast to the pulse pressure, increasing with rising blood pressure. Both arterial pulse pressure and heart rate remained fairly constant during changes in SAP (table 2) and cannot therefore account for the differences in $\Delta \mathrm{V}$.



Fig 10 Effects of arterial hypotension and hypertension on relationship between CSF pulse pressure and ventricular fluid pressure $(V F P)$ in single animal. Three mean $( \pm S D)$ blood pressure levels were: $140.2 \pm 5.6 \mathrm{~mm} \mathrm{Hg}, 90.0 \pm$ $5.4 \mathrm{~mm} \mathrm{Hg}$, and $174.9 \pm 10.1 \mathrm{~mm} \mathrm{Hg}$. Corresponding arterial pulse pressures are shown. Only data below break points were analysed. Linear relationships were found with following regression equations: $y=0.05 x+0.4(r=0.90, p<0.001)$ for normotension, $y=0.14 x-0.5(r=0.95, p<0.001)$ for hypotension, and $y=0.03 x+0.4(r=0.97, p<0.001)$ for hypertension. The slopes were significantly different from each other (F test, $p<0.01)$. Above break points pulse pressures seem to converge.

Table 3 Mean regression equations of relationships between volume-pressure response (VPR) and ventricular fluid pressure (VFP) and between CSF pulse pressure and VFP during systemic arterial normotension, hypotension, and hypertension

\begin{tabular}{|c|c|c|}
\hline & $V P R-V F P$ & $C S F$ pulse pressure-VFP \\
\hline $\begin{array}{l}\text { Normotension } \\
\text { Hypotension } \\
\text { Hypertension }\end{array}$ & $\begin{array}{l}y=0.08 x+0.2 \\
y=0.06 x+0.7 \\
y=0.09 x+0.2\end{array}$ & $\begin{array}{l}y=0.07 x+0.0 \\
y=0.09 x+0.2 \\
y=0.05 x+0.4\end{array}$ \\
\hline
\end{tabular}

\section{Discussion}

The results of this study obtained at normal levels of carbon dioxide and blood pressure are consistent with those of a previous report. ${ }^{2}$ During cerebral compression caused by steady rate inflation of an extradural balloon both VPR and CSF pulse pressure behave differently on either side of a break point ICP. Their linear relationships with the ICP below the break point confirm the validity of the analytical model implying a monoexponential intracranial volume-pressure curve and a constant transient increase in cerebral blood volume per cardiac cycle. The finding of a constant elastance and 
a more rapid increase of the pulse pressure above the break point supports the concept of a linear volume-pressure model and a progressive increase in $\Delta \mathrm{V}$, once the break point ICP has been passed. The mean break point ICPs of $53.4 \mathrm{~mm} \mathrm{Hg}$ in the first and $55.1 \mathrm{~mm} \mathrm{Hg}$ in the second series do not significantly differ from the earlier reported pressure of $59.8 \mathrm{~mm}$ $\mathrm{Hg}$. The physiological explanation for the occurrence of the break point was previously derived from the concepts on the mechanisms underlying VPR and the change in intracranial volume per cardiac cycle (fig 1). ${ }^{2}$ It was then suggested that the break point is associated with cerebral vasoparesis and failure of $\mathrm{CBF}$ autoregulation.

Our present investigations have yielded arguments in favour of the above explanation. Hypercapnia is known to raise the ICP through an increase in cerebral blood volume produced by cerebral vasodilatation ${ }^{10-12}$. During the initial period of cerebral compression $\mathrm{CO}_{2}$ produced increasingly larger rises in ICP (figs 2 and 3). Here, two opposite effects are operative. The gradual decrease in cerebral perfusion pressure induces an autoregulatory vasodilatation and thus reduces the capacity for further dilatation in response to $\mathrm{CO}_{2}$ stimulus. On the other hand, the $\mathrm{CO}_{2}$ induced increments in CBV cause progressively larger rises in ICP, because of the exponential shape of the volumepressure curve. This last effect apparently is the stronger one up to a pressure corresponding with the break point ICP (fig 3). Above the break point, the $\mathrm{CO}_{2}$ effect on the ICP levelled off and finally disappeared. In view of the explanation for the break point two factors can be held responsible for this phenomenon: the linear nature of the volume-pressure relationships (constant VPR) and the gradual loss of $\mathrm{CO}_{2}$ reactivity of the cerebral vessels due to vasoparesis and subsequently vasoparalysis ${ }^{13}$. This does not conflict with the assumption of failure of autoregulation commencing at the break point, as it is well documented that cerebrovascular reactivity to $\mathrm{CO}_{2}$ is retained when autoregulation is abolished. ${ }^{13-16}$ The significantly higher break point ICP during hypercapnia compared with normocapnia may be related to the increased blood pressure level. The autoregulatory constrictor stimulus induced by raised SAP increases the residual vasodilator capacity thus delaying maximal vasodilatation with consequent impairment of autoregulation. The break point therefore seems to be related to the cerebral perfusion pressure, rather than to the level of ICP. Further evidence for relating the break point in the relationships of both VPR and CSF pulse pressure to the ICP to failure of autoregulation can be derived from the second series of experiments, in which changes in SAP were induced. Below the break point, variations in SAP had little influence on the ICP (fig 8). This provides indirect evidence that the cerebrovascular reactivity to changes in perfusion pressure was unimpaired. The typical shape of the VFP tracings each time the SAP was raised or lowered (fig $7 a, b$ ) shows that, in spite of the recurrent infliction of arterial hypotension and hypertension, the autoregulatory vasomotor response of the animal preparations was intact. The absence of the response above the break point ICP indicates that the cerebral vessels behaved in a pressure-passive manner to changes in SAP. As a result, the blood pressure variations produced exaggerated changes in ICP (fig 8). These observations are consistent with those of previous reports. ${ }^{12} 17$ It is interesting to note that the break points at arterial hypotension normotension, and hypertension occurred at successively higher intracranial pressures. Again, the explanation follows from the assumption that the break point is related to disturbance of autoregulation: by raising the SAP the lower limit of autoregulation is shifted towards a higher ICP. The perfusion pressures at the break points, however, differ considerably, the break point at hypertension occurring when the perfusion pressure is still relatively high. This is presumably caused by damage to the flow regulating structures as cerebral compression progresses.

Our main aim was to study the effects of changes in $\mathrm{PaCO}_{2}$ and SAP on the amplitude of the CSF pulse during rising ICP. Since we are mainly interested in the CSF pulse pressure in so far as it is a measure of intracranial elastance, which in its turn may be influenced by the variables under investigation, we had to study the intracranial volume-pressure relationships as well. The volume-pressure test was chosen for the evaluation of these relationships, because of its clinical relevance. Hypercapnia did not significantly alter the elastance coefficient $\left(\mathrm{E}_{1}\right)$ in the individual animals, which implies that the shape of the volume-pressure curve remained unchanged. These results are in line with those of Löfgren, ${ }^{8}$ who recorded the volume-pressure curve by rapid infusion into the cisterna magna of dogs with varying degrees of hypercapnia. He found a slight, though not 
significant, decrease of the elastance. Leech and Miller $^{18}$ and Rowed et $a l^{19}$ also demonstrated that hypocapnia, induced by hyperventilation, has no effect on the elastance. However, the group results of the present study, a reduction of $E_{1}$ in five animals, do suggest a slight flattening of the volume-pressure curve. This might have some meaning, if the positive influence on $E_{1}$ of the raised blood pressure at hypercapnia is taken into account, as will be discussed later.

In contract to the effect on the VPR, hypercapnia produced a significant increase in the amplitude of the CSF pulse. This is in agreement with various other reports. ${ }^{35}$ Symon $^{40}$ used the ratio of venous to arterial pulse height (pulse index) in the pial circulation within the middle cerebral arterial field to assess regional vascular reactivity. The author found an increased pulse index in response to increased $\mathrm{PaCO}_{2}$. Since the venous pulsations in particular are easily transmitted to the CSF, these observations probably also hold for the CSF pulse. It follows from the experimental design of the present study that the increased pulse height is a pure $\mathrm{CO}_{2}$ effect, not related to the $\mathrm{CO}_{2}$ induced rise in ICP. As the elastance coefficient remained unaltered, the explanation for the increase in pulse pressure should, according to the analytical model, be sought in an increase in magnitude of the change in CBV per cardiac cycle during hypercapnia. The $\mathrm{CO}_{2}$ induced cerebral vasodilatation reduces the arterial inflow resistance. On account of the "easier" inflow of blood the time lag between inflow and outflow increases, resulting in a larger $\Delta \mathrm{V}$ (fig 1). An additional factor may be a simultaneous change in the venous outflow profile, because of the slightly raised central venous pressure. Apart from the timing mechanism, the $\mathrm{CO}_{2}$ induced increase in $\mathrm{CBF}$ per se should cause a larger change in $\mathrm{CBV}$ per cardiac cycle, so long as the heart rate remains constant. At the same time, this explains why hypercapnia did not produce a significant effect on the increase in $\Delta \mathrm{V}$, and consequently on the increase in CSF pulse pressure, above the break point, where vasoparesis has occurred and $\mathrm{CO}_{2}$ gradually looses its vasodilatory action. This is clearly demonstrated by fig 6 . The gradients of the relationships between CSF pulse pressure and VFP above the break point are not significantly different, implying an equal increase in $\Delta \mathrm{V}$ at normocapnia and at hypercapnia. Moreover, the break point is virtually absent in the hypercapnic relationship. This is because under hypercapnic conditions the cerebral vessels are widely dilated all the time and the increase in pulse pressure, when autoregulation becomes impaired, is therefore less marked than during normocapnia.

The reason why $\Delta \mathrm{V}$ steadily increases above the break point is that the venous outflow resistance continues to increase because of the so-called cuff constriction of the cerebral veins at their junction with the dural sinuses $^{21-23}$ as well as because of compression of the sinuses themselves. ${ }^{24}$ As a result, the cerebral arterial inflow and venous outflow during a cardiac cycle separate into two processes causing a progressively larger change in CBV (fig 1b). It might be argued that also below the break point $\Delta \mathrm{V}$ is not truly constant, as the inflow resistance is gradually reduced as part of the autoregulatory process. In that case the relationship between pulse pressure and ICP should be non-linear and, indeed, such an approximation might well have fitted the data of this study, as was also shown by Guinane. ${ }^{26}$ However, the effect of the decrease in cerebrovascular resistance on $\Delta \mathrm{V}$ below the break point is apparently so small that the description of the relationship by means of a linear function, and consequently a constant $\Delta \mathrm{V}$, is also valid.

The small rise in arterial pulse height during hypercapnia may be a contributory factor to the increase in CSF pulse pressure but cannot account for the whole phenomenon, as it was also present in animals with only a minimal difference in arterial pulse pressure (table 1). On the other hand, the concomitantly increased blood pressure level tends to reduce the CSF pulse pressure, as will be discussed next, and is thus in competition with the effect of the arterial pulse. Above the break point, however, under conditions of vasoparesis, the increased arterial pulse height may have been responsible for the constant difference in $\Delta \mathrm{V}$ and consequently in CSF pulse pressure between normocapnia and hypercapnia (fig 6). Moreover, the slight increase in perfusion pressure causes a rise in $\mathrm{CBF}$ resulting in a larger $\Delta \mathrm{V}$, as the heart rate remained constant.

In contrast to changes in arterial carbon dioxide tension, variations in arterial blood pressure did affect the intracranial volumepressure relationships, as has been reported by others. $^{67}$ Raising the SAP from hypotensive to hypertensive levels increased the elastance coefficient by aproximately $50 \%$, which is in agreement with the findings of the earlier mentioned investigators, if the same SAP range is considered. Looking at the individual animals, 
however, it was either hypotension or hypertension which produced a significant change in $\mathrm{E}_{1}$ compared to normotension. Only when the extremes, hypotension and hypertension, were compared was a significant difference found in every animal. This implies that in the blood pressure range encountered clinically minor changes in SAP do not necessarily produce a noticeable effect on brain elastance.

The influence of SAP on the elastance can be understood by considering the underlying mechanisms of the VPR. A rapid volume addition to the CSF compartment causes a compression of the venous outflow section and thus a corresponding increase in the outflow resistance. The outflow of blood will consequently be reduced, whereas the inflow remains unaffected, resulting in an increase in CBV with a corresponding rise in CSF pressure. In order that the equilibrium between inflow and outflow be restored the CSF pressure rises to the extent that the perfusion pressure over the inflow tract is decreased proportionally to the increase in outflow resistance. For this reason, the CSF pressure shows a larger rise at higher arterial pressures. The height of the VPR seems therefore to be primarily related to the perfusion pressure over the inflow section of the vascular bed and not to the inflow resistance and the CBF. This may explain why $\mathrm{CO}_{2}$, increasing $C B F$ by reducing the inflow resistance, does not significantly affect the VPR. These arguments seemingly contradict the findings of Leech and Miller, ${ }^{7}$ who described a significant positive correlation between SAP and VPR as well as between CBF and VPR. Once intracranial hypertension had been establised, however, their experimental animals showed failure of autoregulation and the CBF thus responded in a pressure-passive manner to changes in SAP. Although we did not measure the CBF, we assume, for reasons mentioned above, that autoregulation was intact up to the break point ICP.

The results of the current study show that, by contrast to the effect on the VPR, the level of SAP is inversely related to the height of the CSF pulse (table 3). The reason for this must be a decrease in the transient change in CBV per cardiac cycle with rising SAP, since according to the analogy between VPR and CSF pulse pressure an increase in pulse height with rising SAP would have been expected. Thus, during rising SAP two opposite forces are effective on the amplitude of the CSF pulse: an increase of the elastance coefficient and a decrease in $\Delta \mathrm{V}$.
The effect on $\Delta \mathrm{V}$ apparently prevails, as in most animals the CSF pulse pressure significantly decreased when SAP was raised from hypotensive over normotensive to hypertensive levels and vice versa. These findings cannot be explained by changes in the arterial pulse height, since $\triangle$ SAP was relatively little affected when SAP was altered (table 2). Besides, the small changes that occurred were mostly into the same direction as the blood pressure, so that their influence on the CSF pulse pressure was opposite to that of the blood pressure. The arterial pulse pressure is one of the factors determining the pulsatile inflow profile and consequently affects the CSF pulse pressure through the mechanism of $\Delta \mathrm{V}$. The difference in mean $\Delta \mathrm{V}$ between arterial normotension in this series and normocapnia in the first series can therefore be explained by the difference in $\triangle$ SAP.

The explanation of the SAP-induced changes in $\Delta \mathrm{V}$ follows the same argument as outlined above. The alterations in cerebral perfusion pressure elicit an autoregulatory response consisting of vasodilatation during hypotension and vasoconstriction during hypertension with corresponding changes in the inflow resistance and the shape of the pulsatile inflow curve, thus causing an increase and a reduction in $\Delta \mathrm{V}$ respectively (fig 1 ). Fig 10 shows that the difference in CSF pulse height between the various SAP levels tends to disappear above the break point, where cerebral vasoreactivity becomes impaired. Contrary to the results of this study, Hamer et $a l^{5}$ described an increase in CSF pulse height with rising SAP. From their tracings, however, it appears that this was due to a concurrent rise both in ICP and in arterial pulse pressure. Moreover, presumably the increase in SAP did not last long enough for the autoregulatory vasoconstrictor response to develop, so that $\Delta \mathrm{V}$ was not negatively influenced. It might still be argued that the vasomotor response, as shown in the ICP tracings (fig $7 a, b$ ) was not a true autoregulatory response, but merely the result of a direct action of angiotensin and trimethaphan on the cerebral vasculature and that consequently the present results hold true only for drug-induced alterations in SAP. This criticism can be answered by referring to the previously mentioned work of Symon ${ }^{4}$ with regard to the pulse transmission in the middle cerebral field. Lowering the input arterial pressure by exsanguination or temporarily occluding the carotid vessels in the neck caused reduction in pial arterial pressure but a definite increase in the pulse index, which is consistent with an increase 
in CSF pulse pressure. From comparison between intracarotid and intravenous infusion of angiotensin in patients various authors ${ }^{27}{ }^{28}$ have concluded that the increase in cerebrovascular resistance was secondary to the increase in blood pressure, representing autoregulation, and not the result of a direct effect on the cerebral vessels. The same was demonstrated for trimethaphan. ${ }^{29}$ Finally, additional evidence for the vasomotor response representing true autoregulation is found by studying the time lag between the change in SAP and the change in ICP in the opposite direction (fig $7 a, b$ ). This was always in the order of 10 to 30 seconds, which is in agreement with the reported time intervals necessary for autoregulation to adjust. ${ }^{30-33}$

This study was directed toward clinical applicability. The idea of using the CSF pulse pressure to ICP ratio as a continuous parameter of intracranial volume-pressure relationships was first launched by two of us in a preliminary clinical report ${ }^{1}$ and subsequently further developed in an extensive experimental study. ${ }^{2}$ However, in both patients and experimental animals, the gradient of the relationship between pulse pressure and ICP did not correlate with the slope of the volume-pressure curve as defined by the elastance coefficient. This was not surprising, as it had been mathematically demonstrated that the gradient of the relationship also depends on the magnitude of the change in $C B V$ per cardiac cycle. Since $\Delta V$ shows a biological variation between individuals, their volume-pressure relationships cannot be accurately assessed on the basis of the CSF pulse pressure alone. In the same individual, however, $\Delta \mathrm{V}$ has been shown to remain constant when ICP rises, provided that autoregulation is maintained. This has important implications for the clinical practice of ICP monitoring. At the beginning of a pressure recording the elastance coefficient can be calculated on the basis of a few volume-pressure tests. Thereafter, any change in the CSF pulse pressure to ICP ratio, which can be monitored by means of on-line computer analysis, indicates a change in elastance coefficient. This, of course, holds true only so long as the condition of the patient is stable with regard to both intracranial and extracraniel haemodynamics, since they will, through the mechanism of $\Delta \mathrm{V}$, also affect the CSF pulse pressure. It has already been shown that loss of autoregulation and sudden cerebral vasodilatation, such as those occurring during plateau waves, causes an abrupt increase in pulse pressure. ${ }^{12}$ This study has evaluated the influence on $\Delta \mathrm{V}$ of some other major factors: arterial $\mathrm{CO}_{2}$ tension, blood pressure, arterial pulse pressure, and heart rate. Since these variables are routinely monitored in patients in whom continuous ICP recording is indicated, information on variations in $\Delta \mathrm{V}$ will be constantly available. This may help to interpret changes in the slope of the relationship between CSF pulse pressure and ICP in terms of changes in intercranial volume-pressure relationships.

This study was supported by a grant from the Netherlands Organisation for the Advancement of Pure Research (ZWO). We are grateful to the staffs of the Institute of Neurological Sciences and the Wellcome Surgical Research Institute, Glasgow, for their assistance.

\section{References}

1 Avezaat CJJ, van Eijndhoven JHM, de Jong DA, Moolenaar WCJ. A new method of monitoring intracranial volume-pressure relationships. In: Beks JFW, Bosch DA, Brock M, eds. Intracranial Pressure III. Berlin: Springer, 1976: 308-13.

2 Avezaat CJJ, van Eijndhoven JHM, Wyper DJ. The cerebrospinal fluid pulse pressure and the intracranial volume-pressure relationships. $J$ Neurol Neurosurg Psychiatry 1979; 42:687-700.

3 Goldensohn ES, Whitehead RW, Parry TM, Spencer JN, Grover RF, Draper WB. Studies on Diffusion Respiration. IX. Effect of Diffusion Respiration and High Concentrations of $\mathrm{CO}_{2}$ on Cerebrospinal Fluid Pressure of Anesthetized Dogs. Am J Physiol 1951; 165:334-40.

4 Symon L. Regional vascular reactivity in the middle cerebral arterial distribution. An experimental study in baboons. J Neurosurg 1970; 33:532-41.

5 Hamer J, Alberti E, Hoyer S, Wiedemann K. Influence of systemic and cerebral vascular factors on the cerebrospinal fluid pulse waves. J Neurosurg 1977; 46:35-45.

6 Löfgren J. Effects of variations in arterial pressure and arterial carbon dioxide tension on the cerebrospinal fluid pressure-volume relationships. Acta Neurol Scand 1973; 49:586-98.

7 Leech P, Miller JD. Intracranial volumepressure relationships during experimental brain compression in primates. 3. Effect of mannitol and hyper-ventilation. $J$ Neurol Neurosurg Psychiatry 1974; 37:1105-111.

8 Miller JD, Garibi J. Intracranial volume-pressure relationships during continuous monitoring of ventricular fluid pressure. In: Brock M, Dietz $H$, eds. Intracranial Pressure. Berlin: Springer, 1972; 270-4. 
9 Severinghaus JW. Blood gas calculator. J Appl Physiol 1966; 21:1108-16.

10 Wolff HG, Lennox WG. The effect of pial vessels of variations in the oxygen and carbon dioxide content of the blood. Arch Neurol Psychiatry 1930; 23:1097-120.

11 Reivich M. Arterial $\mathrm{pCO}_{2}$ and cerebral hemodynamics. Am J Physiol 1964; 206:25-35.

12 Langfitt TW, Kasell NF, Weinstein JD Cerebral blood flow with intracranial hypertension. Neurology 1965; 15:761-73.

13 Langfitt TW, Weinstein JD, Kassell NF. Cerebral vasomotor paralysis produced by intracranial hypertension. Neurology (Minneap) 1965; 15: 622-41.

14 Häggendal E, Johannson B. Effects of arterial carbon dioxide tension and oxygen saturation on cerebral blood flow autoregulation in dogs. Acta Physiol Scand 1965; 66: suppl 258, 27-53.

15 Ekström-Jodal $B$. On the relation between blood pressure and blood flow in the canine brain with particular regard to the mechanism responsible for cerebral blood flow autoregulation. Acta Physiol Scand 1970; suppl 350:5-61.

16 Miller JD, Stanek AE, Langfitt TW. Cerebral blood flow regulation during experimental brain compression. J Neurosurg 1973; 39:186-96.

17 Harper AM. Autoregulation of cerebral blood flow: influence of arterial blood pressure on the blood flow through the cerebral cortex. $J$ Neurol Neurosurg Psychiatry 1966; 29:398-403.

18 Leech P, Miller JD. Intracranial volume-pressure relationships during experimental brain compression in primates. 2 . Effect of induced changes in systemic arterial pressure and cerebral blood flow. J Neurol Neurosurg Psychiat 1974; 37:1099104.

19 Rowed DW, Leech PJ, Reilly PL, Miller JD. Hypocapnia and intracranial volume-pressure relationship. Arch Neurol 1975; 32:369-73.

20 Symon L. Transmission of arterial pulsations in the cerebral vascular bed of the baboon. $J$ Physiol 1968; 196:52P-53P.

21 Wright RD. Experimental observations on increased intracranial pressure. Aust NZ J Surg 1938; 7-8:215-35.

22 Bedford THB. The effect of variations in the subarachnoid pressure on the venous pressure in the superior longitudinal sinus and in the torcular of the dog. $J$ Physiol 1942; 101:362-8.

23 Hedges TR, Weinstein JD, Kassell N, Stein S. Cerebrovascular responses to increased intracranial pressure. J Neurosurg 1964; 21:292-7.

24 Langfitt TW, Weinstein JD, Kassell NF, Gagliardi, LJ, Shapiro HM. Compression of cerebral vessels by intracranial hypertension. I. Dural sinus pressures. Acta Neurochir 1966; 15:212-22.

25 Shapiro JH, Langfitt TW, Weinstein JD. Compression of cerebral vessels by intracranial hypertension. II. Morphological evidence for collapse of vessels. Acta Neurochir 1966; 15: 222-33.

26 Guinane FE. Cerebrospinal fluid pulse pressure and brain compliance in adult cats. Neurology (Minneap) 1975; 25:559-64.

27 Greenfield JC, Tindall GT. Effect of norepinephrine, epinephrine and angiotensin on blood flow in the internal carotid artery of man. $J$ Clin Invest $1968 ; 47: 1672-84$.

28 Oleson J. The effect of intracarotid epinephrine, norepinephrine and angiotensin on the regional cerebral blood flow in man. Neurology (Minneap) 1972; 22:978-87.

29 Oleson J. Quantitative evaluations of normal and pathologic cerebral blood flow regulation to perfusion pressure. Changes in man. Arch Neurol (Chicago) 1973; 28:143-9.

30 Rapela CE, Green HD. Autoregulation of Canine Cerebral Blood Flow. Circ Res 1964; 15: suppl. 1, 205-12.

31 Kanzow E. Diskussion in Pharmakologie der lokalen Gehirndurchblutung. Messmethoden und Ergebnisse. In: Betz E, Wüllenweber $\mathbf{R}$, eds. Ein internationales Symposium, Bonn: Aertzliche Forschungen. Beiheft, 1968: 177-8.

32 Ekström-Jodal B, Häggendal E, Nilsson NJ. Cerebral venous oxygen saturation during rapid changes in the arterial blood pressure. An oximetric study in dogs. Acta Physiol Scand 1970; suppl. 350:43-50.

33 Symon L, Held K, Dorsch NWC. On the myogenic nature of the autoregulatory mechanism in the cerebral circulation. Part 1. Eur Neurol 1972; 6:11-18. 